\title{
Abuse Potential of Intravenous Oxycodone/Naloxone Solution in Nondependent Recreational Drug Users
}

\author{
Salvatore V. Colucci • Peter J. Perrino • \\ Megan Shram · Cynthia Bartlett · Yi Wang • \\ Stephen C. Harris
}

Published online: 23 April 2014

(c) The Author(s) 2014. This article is published with open access at Springerlink.com

\begin{abstract}
Background and Objective Abuse of opioid analgesics has become a public health issue. Some opioid abusers use intravenous administration to increase the magnitude of positive reinforcing effects. Intravenous co-administration of oxycodone with naloxone, an opioid antagonist, may reduce these rewarding effects and discourage abuse. A 2:1 oxycodone:naloxone $(\mathrm{OXN})$ tablet formulation has been studied in the USA for the management of moderate-tosevere chronic pain. Intravenous administration of a 2:1 oxycodone:naloxone solution ( $\mathrm{sOXN}$ ) reflects the oxycodone:naloxone ratio found in laboratory studies of OXN following tampering for intravenous administration. The objective of this study was to characterize abuse-deterrent properties of sOXN.

Methods This single-center, double-blind, randomized, placebo-controlled, active-controlled, crossover study enrolled nondependent recreational opioid users with experience using multiple (two or more) routes of administration. Following demonstration that subjects could discern between placebo and oxycodone, 24 eligible male and female subjects were randomized to receive intravenous injections of $0.07 \mathrm{mg} / \mathrm{kg}$ oxycodone (OXY), $0.07 \mathrm{mg} /$
\end{abstract}

S. V. Colucci · P. J. Perrino - Y. Wang · S. C. Harris ( $\square)$ Purdue Pharma L.P., One Stamford Forum, 201 Tresser Blvd, Stamford, CT 06901, USA

e-mail: Stephen.Harris@pharma.com

M. Shram · C. Bartlett

INC Research Toronto, 720 King Street West, Toronto, ON, Canada $\mathrm{kg}$ oxycodone and $0.035 \mathrm{mg} / \mathrm{kg}$ naloxone solution (sOXN), or matching placebo over three visits. Pharmacokinetics, pharmacodynamics, safety, and tolerability were assessed at scheduled times up to $8 \mathrm{~h}$ post-dose. Parameters were computed and statistically compared among treatments. Results Pharmacokinetics were similar between OXY and sOXN. Subjects reported significantly fewer rewarding effects with sOXN compared with OXY; differences between sOXN and placebo were generally not significant. sOXN was well tolerated.

Conclusions Significant reductions in drug liking and other subjective effects following administration of sOXN compared with OXY indicate that naloxone concentrations were sufficient to antagonize the effects of oxycodone when abused by the intravenous route of administration in opioid-experienced drug users.

\section{Key Points}

Significant reductions in the degree of drug liking and other pharmacodynamic effects following intravenous administration of oxycodone/naloxone compared with oxycodone were consistent with the agonist-antagonist interaction hypothesis.

Naloxone concentrations were sufficient to antagonize the reinforcing effects of oxycodone when abused by the intravenous route of administration in opioid-experienced drug users.

Drug liking reduction by intravenous administration of oxycodone/naloxone observed in this study may not represent the situation in the real-world settings. 


\section{Introduction}

Extended-release opioid analgesics are approved for the treatment of moderate-to-severe pain with less frequent dosing than immediate-release opioid analgesics [1]. A significant public health challenge is to ensure access to these products while reducing their misuse and abuse [2, 3]. Misuse and abuse of analgesics has numerous adverse healthcare implications, including psychiatric illness, that increase the cost burden to insurers [3, 4].

Abusers manipulate opioid formulations to obtain faster absorption, which can produce a state of euphoria or "high" [5]. For opioid analgesics, illicit administration can occur by nasal insufflation of crushed pills and intravenous administration of tablets dissolved in an aqueous medium [6]. Oxycodone is an opioid analgesic that has been associated with manipulation for injection by recreational drug users, with needle sharing increasing the health risk $[6,7]$.

Abuse-deterrent strategies include incorporating physical and chemical barriers, formulating agonist/antagonist combinations, adding aversive agents, modifying the delivery system, developing prodrugs, or using combinations of these techniques [2]. However, it is important that the intended analgesic efficacy of the medication be maintained regardless of the abuse-deterrent strategy employed [2].

A combination tablet formulation with controlledrelease oxycodone hydrochloride and naloxone (2:1 ratio) is currently marketed in 29 countries and has been studied in the USA for the management of moderate-to-severe chronic pain. A previous study determined that oxycodone/naloxone at a 2:1 ratio had optimal efficacy and safety compared with other ratios [8]. Naloxone is a competitive opioid receptor antagonist with low systemic activity when administered by the oral route because of low oral bioavailability ( $\leq 2 \%$ ) [9]. In contrast, intravenous administration results in high plasma concentrations of naloxone, suggesting that the intravenous administration of this formulation could block the agonist activity of oxycodone, thereby conferring abuse-deterrent properties [10].

The purpose of the current study was to evaluate the abuse potential, pharmacodynamics, pharmacokinetics, and safety and tolerability of intravenous administration of oxycodone with naloxone (2:1 ratio) in nondependent, opioid-experienced, recreational drug users consistent with recommendations in the US Food and Drug Administration's (FDA's) recent draft guidance [2]. The pharmacokinetics and pharmacodynamics following intravenous coadministration of oxycodone and naloxone were characterized to evaluate the abuse-deterrent properties of this agonist/antagonist formulation in an opioid-experienced, nonphysically dependent population.

\section{Methods}

\subsection{Study Design}

This single-center, double-blind, randomized, placebocontrolled, active-controlled, crossover study was conducted at INC Research Toronto, Toronto, Ontario, Canada in accordance with the Declaration of Helsinki and its amendments as outlined by the International Conference on Harmonisation. Prior to the initiation of the clinical study, the protocol, consent form, amendments to the protocol, and advertisements for the recruitment of subjects were reviewed and approved by the Ontario Institutional Review Board in Aurora, Ontario, Canada. All subjects provided written, informed consent prior to the initiation of any study-related procedures.

This study comprised four phases: screening, qualification, treatment, and follow-up. The screening phase was to confirm that subjects had moderate experience with abuse of opioids but were not currently physically opioid dependent, consisting of two visits: visit 1 occurred within 21 days of first administration of study drug, and visit 2 (naloxone challenge test [11]) took place within at least $12 \mathrm{~h}$ prior to the qualification phase. In this test, subjects received an initial dose of $0.2 \mathrm{mg}$ naloxone $\mathrm{HCl}$ intravenously followed by a subsequent dose of $0.6 \mathrm{mg}$ if no evidence of withdrawal (Objective Opiate Withdrawal Scale [12] score of $\geq 3$ ) was observed.

The qualification phase, 3 days in duration, was to determine whether candidate subjects could (a) tolerate the effects of oxycodone and (b) discriminate between the active compound and placebo. Randomized subjects received either a single intravenous dose of oxycodone $0.07 \mathrm{mg} / \mathrm{kg}$ (OXY) or matching placebo with a washout of $24 \mathrm{~h}$. Qualified subjects adequately distinguished between OXY and placebo [a difference of at least 15 points (30\%) greater on the 100-point bipolar (i.e., $50=$ neutral response) on a Drug Liking visual analog scale (VAS), and at least 10 points $(20 \%)$ greater on the 100-point bipolar Overall Drug Liking VAS for OXY compared with placebo]; and tolerated $0.07 \mathrm{mg} / \mathrm{kg}$ oxycodone.

The treatment phase consisted of three visits, each lasting 2 days with one overnight stay. During the treatment phase, subjects received each of the study drugs by intravenous administration in a randomized, double-blind fashion. A follow-up phase completed the study, with subjects returning for one visit, which was scheduled 3-7 days after the last administration of the study drug in the treatment phase.

\subsection{Subjects}

The study population consisted of healthy male and female subjects, aged 18-55 years, who were nondependent 
recreational drug users and experienced with multiple routes (two or more) of opioid administration. Main inclusion criteria were a body mass index (BMI) of $18.0-29.9 \mathrm{~kg} / \mathrm{m}^{2}$ with a minimum weight of $50 \mathrm{~kg}$. Candidate subjects must have taken a dose equivalent to $40 \mathrm{mg}$ oxycodone by any route of administration at least one time during their lifetime. A negative urine drug test was required prior to the naloxone challenge and all treatment visits. Candidates were not excluded from the study if cannabinoids and benzodiazepines were present in their system because of the long half-life of the parent molecule or metabolites. However, urine drug tests for these molecules were required to be negative, stable, or decreasing during the study. Negative breath alcohol tests were required at all visits.

Main exclusion criteria included symptoms of withdrawal on the naloxone challenge test (Objective Opiate Withdrawal score $\geq 3$ ) or a self-reported history of dependence within the previous 2 years, or participation in a rehabilitation program at any time. Subjects were also excluded if they had any clinically relevant findings in their medical history, including a history or presence of any significant illness, past or planned abdominal surgery, a history of hypotension, or acute asthma or other obstructive airway disease. Additionally, candidates were excluded for consuming $>20$ cigarettes per day prior to screening or being unable to abstain from smoking for at least $10 \mathrm{~h}$. Use of nonprescription medications, prescription medications, or natural health products was not permitted, except acetaminophen ( $\leq 2 \mathrm{~g} /$ day), vitamin or mineral supplements, acceptable forms of birth control, or hormone replacement within 7 days of the first dose of study drug or throughout the study.

\subsection{Treatment}

Subjects received each of the following intravenous treatments per visit: (1) oxycodone $0.07 \mathrm{mg} / \mathrm{kg}$ + naloxone $0.035 \mathrm{mg} / \mathrm{kg}$ solution (sOXN; Purdue Pharma LP, Wilson, NC, USA); (2) oxycodone $0.07 \mathrm{mg} / \mathrm{kg}+$ naloxone placebo (OXY); and (3) placebo (oxycodone placebo + naloxone placebo; Bard Pharmaceuticals Ltd, Cambridge, UK). The investigational products used in the study included: OxyNorm ${ }^{\circledR}$ (oxycodone hydrochloride; NAPP Pharmaceuticals, Cambridge, UK) $10 \mathrm{mg} / \mathrm{mL}$; saline $(0.9 \% \mathrm{NaCl})$; and naloxone hydrochloride $1 \mathrm{mg} / \mathrm{mL}$ (Sandoz, Montréal, QC, Canada).

Oxycodone $0.07 \mathrm{mg} / \mathrm{kg}$ was selected as the study dose to produce detectable and significant drug effects in recreational drug users with tolerance to opioids than an opioid-naïve population [13]. Naloxone $0.035 \mathrm{mg} / \mathrm{kg}$ was selected to simulate the $2: 1$ ratio that is intended for the oxycodone/naloxone formulation. Naloxone or placebo was administered as a bolus injection followed by infusion of oxycodone or placebo within $1 \mathrm{~min}$.

\subsection{Pharmacokinetics and Pharmacodynamics}

Pharmacokinetic and pharmacodynamic assessments were performed over $8 \mathrm{~h}$ post-dose. Blood samples were collected pre-dose and 5 min, $0.25,0.5,0.75,1,1.5,2,3,4,6$, and $8 \mathrm{~h}$ after treatment administration. Plasma samples were prepared within $60 \mathrm{~min}$ of blood draw by centrifugation $\left(3,000 \mathrm{rpm}, 4{ }^{\circ} \mathrm{C}, 10 \mathrm{~min}\right)$ and stored at $-20{ }^{\circ} \mathrm{C}\left( \pm 5{ }^{\circ} \mathrm{C}\right)$ until analysis. The following parameters were estimated: time to peak exposure $\left(t_{\max }\right)$; terminal elimination half-life $\left(t_{1 / 2}\right)$; peak exposure $\left(C_{\max }\right)$; and area under the concentration-time curve (AUC). Subjective pharmacodynamic measurements were conducted at pre-dose (except for measures specifically assessing drug effects) and $5 \mathrm{~min}, 0.25,0.5$, $0.75,1,1.5,2,3,4,6$, and $8 \mathrm{~h}$ after treatment administration; Subjects were asked to quantify treatment effects on 100-point VAS that were either unipolar (i.e., $0=$ no effect) or bipolar (i.e., $50=$ neutral response). Bipolar scales included Drug Liking, Overall Drug Liking, Take Drug Again, and Alertness/Drowsiness. Unipolar measurements included: High, Good Effects, Bad Effects, and Any Effects.

Subjective drug value was determined by asking subjects to make a hypothetical choice between receiving another dose of sOXN and receiving a specified amount of money (US\$0.25-\$50.00). Depending on the answer to the question, follow-up questions determined the crossover point at which the subject was indifferent about choosing the drug or choosing the money. Subjects knew that they would not actually receive another dose of drug or the money discussed during this evaluation. This test is adapted from a similar procedure used by Griffiths and colleagues [14, 15].

Pupillometry was assessed as an objective physiological measure of opioid effect, using a pupillometer from NeurOptics Inc. (Irvine, CA, USA).

\subsection{Bioanalytical Assay}

Plasma concentrations of oxycodone were determined by a validated bioanalysis method using liquid chromatography with tandem mass spectrometry (LC/MS/MS) following solid-phase extraction over a concentration range of $0.100-50.0 \mathrm{ng} / \mathrm{mL}$. Plasma concentrations of naloxone were measured by a validated bioanalysis method using LC/MS/MS following liquid-liquid extraction over concentration range of $10.0-5,000 \mathrm{pg} / \mathrm{mL}$. The internal standard for oxycodone was oxycodone-D3 and for naloxone was naloxone-D5. The accuracy and precision of the analytic method were ensured on the basis of the results from each analysis of trial samples. For oxycodone, accuracy averaged $0.500 \%[4.40 \%$ relative standard deviation (RSD)] at $0.200 \mathrm{ng} / \mathrm{mL}, 1.60 \%$ (3.64\% RSD) at $5.00 \mathrm{ng} /$ $\mathrm{mL}$ and $-4.60 \%(3.79 \% \mathrm{RSD})$ at $50.0 \mathrm{ng} / \mathrm{mL}$. For naloxone, accuracy averaged $0.00 \% \quad(6.75 \% \quad$ RSD $)$ at 
$20.0 \mathrm{pg} / \mathrm{mL}, 0.600 \%(4.35 \% \mathrm{RSD})$ at $500 \mathrm{pg} / \mathrm{mL}$ and $0.00 \%(5.90 \% \mathrm{RSD})$ at $5,000 \mathrm{pg} / \mathrm{mL}$.

\subsection{Safety and Tolerability}

Evaluations of safety and tolerability included physical examinations, 12-lead electrocardiogram, continuous cardiac monitoring (e.g., pulse rate, \% oxygen saturation), vital signs, clinical laboratory tests, medication history, and adverse events (AEs). AEs were recorded during the period from the signing of the informed consent form through the follow-up visit. AEs were observed and categorized by volunteered signs or symptoms, on clinical assessment, and through nonleading questions at study visits.

\subsection{Statistical Plan}

Three study populations were defined. The pharmacokinetic population comprised all subjects who received one dose of study drug during the treatment phase and had evaluable pharmacokinetic data. All subjects who completed all visits of the treatment phase and did not have any protocol violations made up the pharmacodynamic population. The safety population included all subjects who received one dose of the study drug during the treatment phase. The sample sizes were not determined on the basis of statistical calculation, but as a suitable sample size based on previous studies of this type [13].

Descriptive statistics were used to summarize all pharmacokinetic and pharmacodynamic data. The pharmacodynamic endpoints for the treatment phase were analyzed with a mixed-effect model for crossover study. For each model, data were computed to determine the means, $95 \%$ confidence intervals, and $\mathrm{p}$ values for treatments and the differences between treatments. The contrasts to assess the abuse potential for sOXN included: $\mathrm{sOXN}$ vs. placebo, OXY vs. placebo, and sOXN vs. OXY. The calculation of the percent reduction in Drug Liking VAS maximum effect $\left(E_{\max }\right)$ between sOXN and OXY was developed based on the responder analysis described in recent guidance from the US FDA [2].

Statistical analyses were performed with $\mathrm{SAS}^{\circledR}$ (release 9.2, SAS Institute, Cary, NC, USA). Pharmacokinetic calculations were completed with WinNonLin ${ }^{\circledR}$ (version 5.2, PharSight Corporation, Sunnyvale, CA, USA).

\section{Results}

\subsection{Disposition and Demographics}

Forty-two subjects were eligible for the qualification phase. Twenty-four of these 42 subjects $(57.1 \%)$ successfully met the criteria for qualification, and were enrolled in the treatment phase. Among these 24 subjects, the mean age was 34.9 years, the proportion of male subjects was $87.5 \%$, and the majority of subjects were white $(87.5 \%)$. All subjects had used opioids in the past with $79 \%$ having used cannabinoids. Other drug use was less prevalent. Twenty-two subjects completed the study.

\subsection{Pharmacokinetics}

Mean observed $C_{\max }$ of oxycodone was $98.6 \mathrm{ng} / \mathrm{mL}$ after sOXN administration and $83.1 \mathrm{ng} / \mathrm{mL}$ after OXY (Fig. 1a). The mean AUC of oxycodone was $116.4 \mathrm{ng} \cdot \mathrm{h} / \mathrm{mL}$ after sOXN and $114.7 \mathrm{ng} \cdot \mathrm{h} / \mathrm{mL}$ after OXY, respectively. Median $t_{\max }$ and median $t_{1 / 2}$ after sOXN vs. OXY were 0.05 and $0.05 \mathrm{~h}$, respectively, and 3.5 and $3.4 \mathrm{~h}$, respectively. After sOXN administration, the mean $C_{\max }$ for naloxone was $25.3 \mathrm{ng} / \mathrm{mL}$, and the naloxone AUC was $12.7 \mathrm{ng} \cdot \mathrm{h} / \mathrm{mL}$
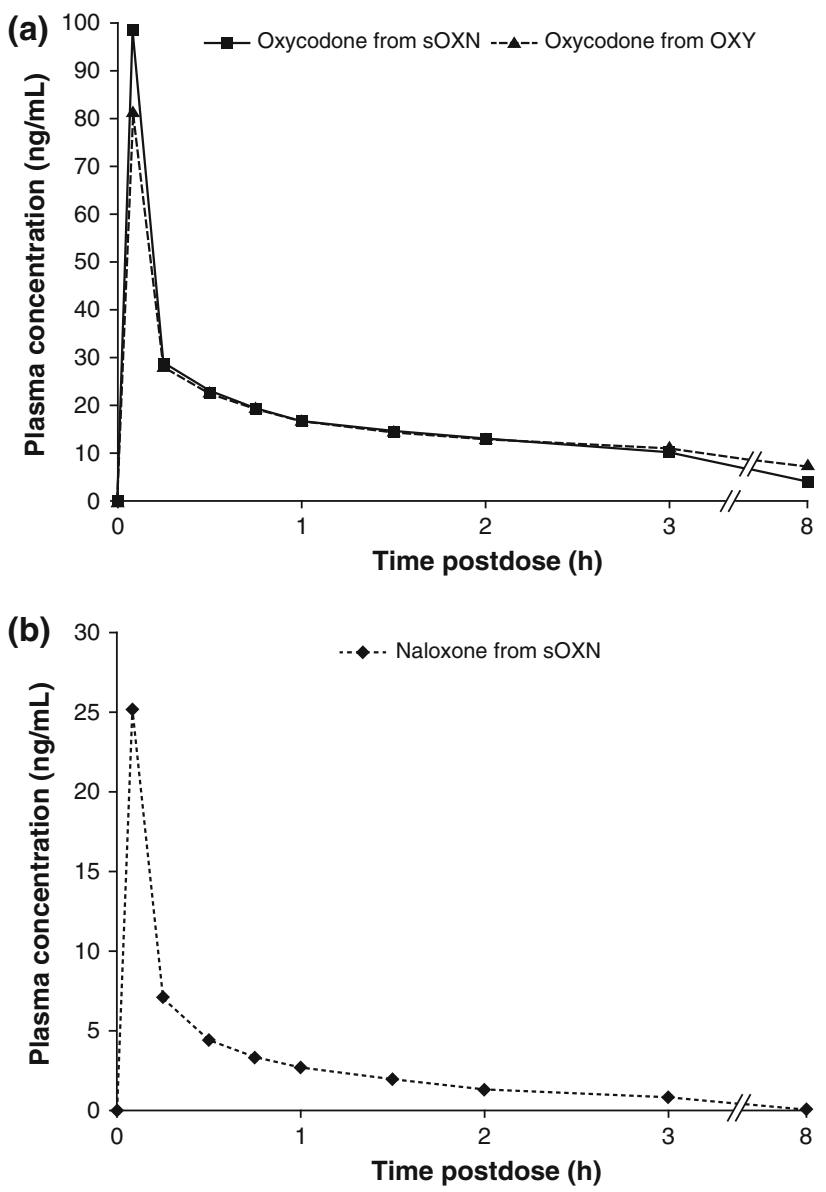

Fig. 1 a Mean plasma oxycodone concentrations $(\mathrm{ng} / \mathrm{mL})$ over time. Oxycodone was administered intravenously as oxycodone $0.07 \mathrm{mg} /$ $\mathrm{kg}+$ naloxone $0.035 \mathrm{mg} / \mathrm{kg}(\mathrm{sOXN} ; n=24)$ or oxycodone $0.07 \mathrm{mg} /$ $\mathrm{kg}+$ placebo (OXY; $n=23)$. b Pharmacokinetics of naloxone $(\mathrm{ng} /$ $\mathrm{mL}$ ) over time. Naloxone was administered intravenously as oxycodone $0.07 \mathrm{mg} / \mathrm{kg}$ + naloxone $0.035 \mathrm{mg} / \mathrm{kg}(\mathrm{sOXN} ; n=24)$ 
Fig. 2 Mean at the Moment Drug Liking VAS scores over time for OXY, sOXN, or placebo $(n=22)$. $s O X N$ simulated oxycodone/naloxone, $O X Y$ oxycodone, $V A S$ visual analog scale

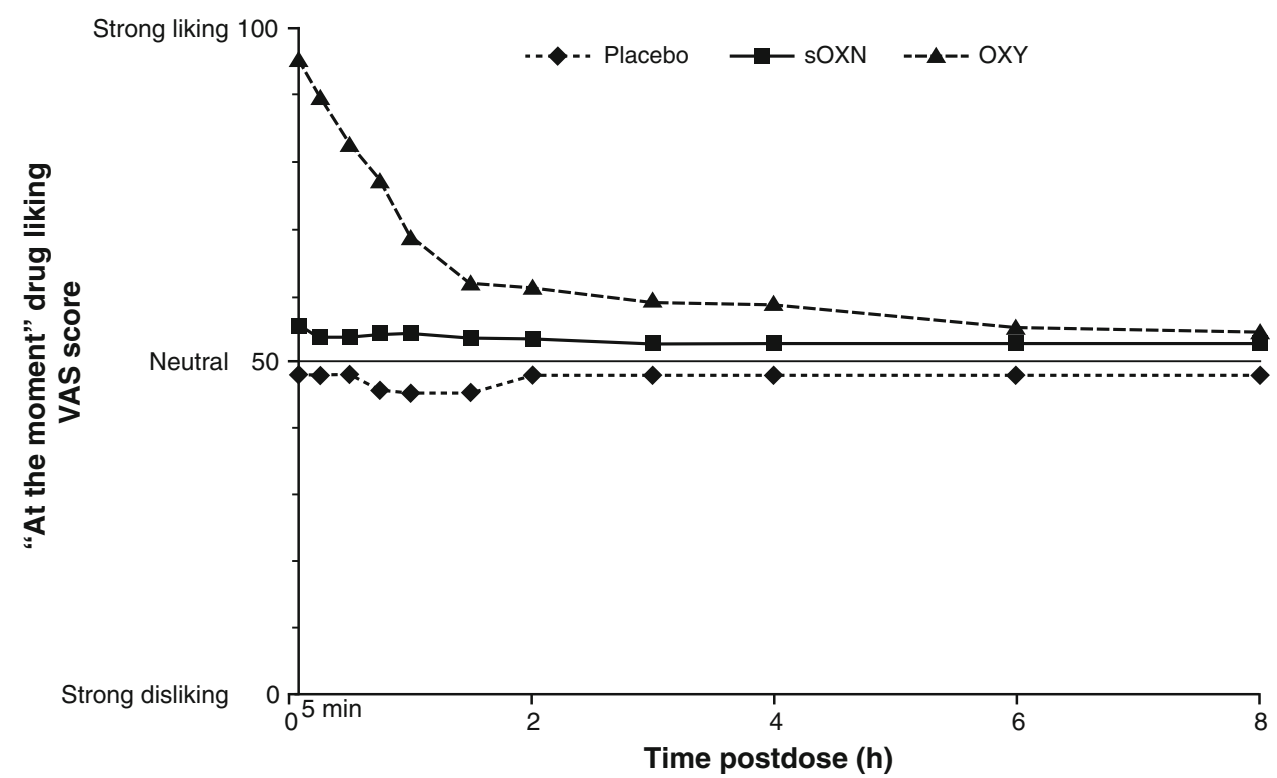

(Fig. 1b). The median $t_{\max }$ for naloxone was $0.05 \mathrm{~h}$ with a median $t_{1 / 2}$ of $1.2 \mathrm{~h}$.

\subsection{Pharmacodynamics}

The mean VAS scores over time for Drug Liking "at this moment" were higher after OXY administration than after sOXN or placebo administration (Fig. 2). The $E_{\max }$ scores were 96.4 for OXY, 56.5 for sOXN, and 48.7 for placebo. The differences in each pairwise comparison were significant: OXY vs. placebo, $p<.001 ;$ sOXN vs. OXY, $p<.001$; and sOXN vs. placebo, $p=.05$.

A responder analysis using $E_{\max }$ of Drug Liking "at this moment" VAS was conducted in 21 subjects who had evaluable data with sOXN and OXY during the treatment phase. In all, $95.2 \%$ of subjects reported a $\geq 50 \%$ reduction in the Drug Liking VAS for sOXN compared with OXY alone (Fig. 3).

In end-of-treatment assessments, the global measures of Overall Drug Liking VAS and Take Drug Again VAS were highest after OXY administration compared with sOXN and placebo (Fig. 4; Table 1). The p values for pairwise differences in these VAS scores were statistically significant for sOXN vs. OXY and OXY vs. placebo $(p \leq .001)$, but not for sOXN vs. placebo. The mean scores for Subjective Drug Value were US\$25.06 for OXY, US\$3.38 for sOXN, and US $\$ 0.86$ for placebo. The differences in value for placebo vs. OXY and sOXN vs OXY were statistically significant $(p<.001)$ but not the difference in placebo vs. $\operatorname{sOXN}(p=.31)$.

Mean Good Effects VAS scores over time are shown in Fig. 5. For $E_{\max }$ scores of Good Effects VAS and Feeling High VAS, the differences for placebo vs. OXY and sOXN vs OXY were statistically significant $(p<.001)$, but there

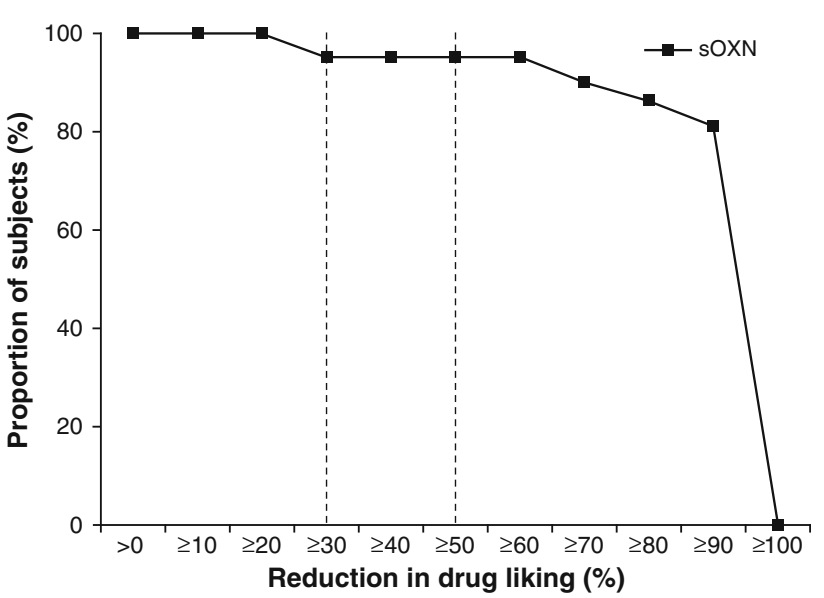

Fig. 3 Percentage of nondependent opioid drug users indicating a reduction in Drug Liking VAS $E_{\max }$ following administration of sOXN $(0.07 \mathrm{mg} / \mathrm{kg}$ oxycodone and $0.035 \mathrm{mg} / \mathrm{kg}$ naloxone $)$ compared with OXY $(0.07 \mathrm{mg} / \mathrm{kg}$ oxycodone; $n=21) . E_{\max }$ maximum effect, sOXN simulated oxycodone/naloxone, $O X Y$ oxycodone

was no significant difference between placebo and sOXN. The mean $E_{\max }$ of Bad Effects VAS and Feeling Sick VAS were low after all treatments and the overall treatment effects for these scores were not significant. The mean $E_{\text {min }}$ of Drowsiness/Alertness VAS was lowest after OXY administration and similar between sOXN and placebo. The differences between OXY and sOXN or placebo were significant $(p<.001$ and $p=.04$, respectively), whereas the difference between sOXN and placebo was not $(p=.91)$.

Mean pupil diameter over time is shown in Fig. 6. After OXY administration, the maximum pupil diameter constriction was $2.8 \mathrm{~mm}$ [standard deviation (SD) $0.7 \mathrm{~mm}$ ]. sOXN-induced miosis was substantially smaller with a 


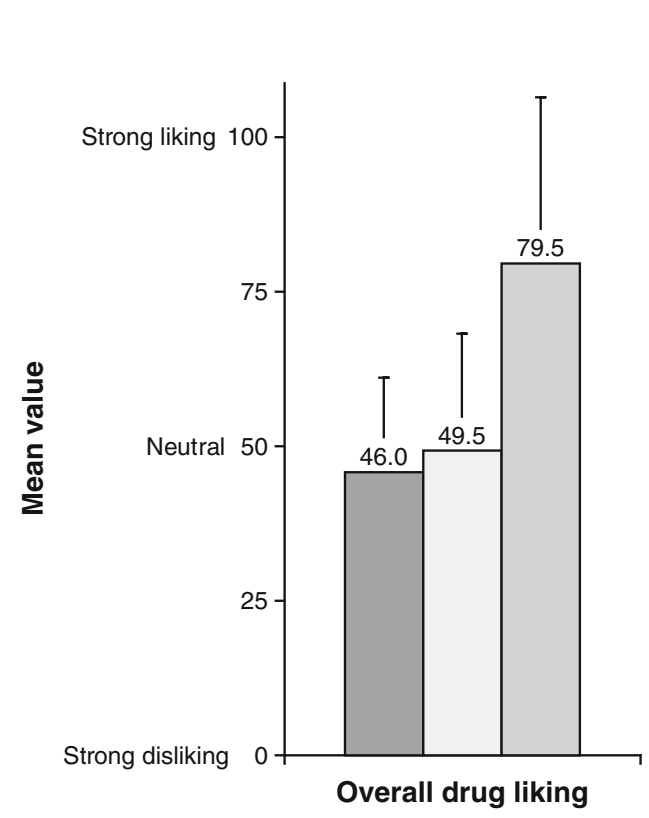

Fig. 4 End-of-treatment balance measures, administered at $8 \mathrm{~h}$ postdose $(n=22)$. Bars indicate mean (SD) of each measure. For Overall Drug Liking VAS (left panel), Take Drug Again VAS (center panel),
Placebo $\square$ sOXN $\square$ OXY
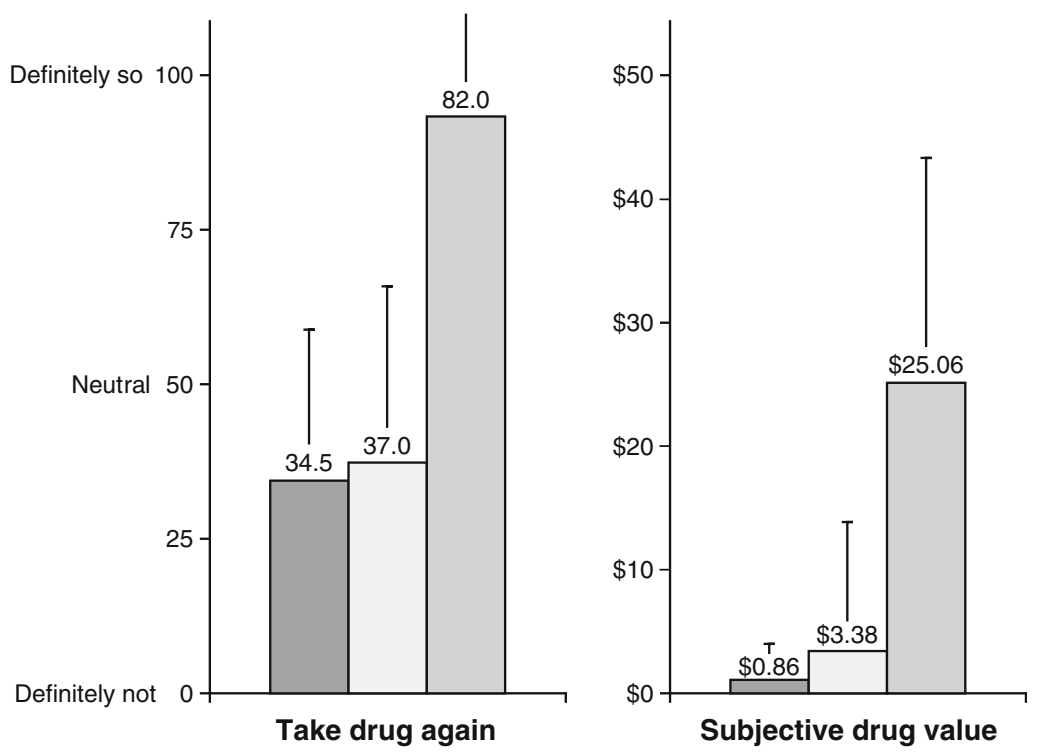

and Subjective Drug Value (right panel), $p<0.001$ for OXY vs. both sOXN and placebo. $s O X N$ simulated oxycodone/naloxone, $O X Y$ oxycodone, $V A S$ visual analog scale, $S D$ standard deviation

Table 1 Summary of VAS scores

\begin{tabular}{|c|c|c|c|c|c|c|}
\hline \multirow[t]{2}{*}{ Parameter } & \multicolumn{3}{|c|}{ Mean (SD) $E_{\max }$} & \multicolumn{3}{|c|}{$p$ value from pairwise comparison } \\
\hline & OXY & sOXN & Placebo & OXY vs sOXN & sOXN vs Placebo & OXY vs Placebo \\
\hline Overall Drug Liking ${ }^{\mathrm{a}}$ & $79.5(26.7)$ & $49.5(18.5)$ & $46.0(15.0)$ & 0.001 & 0.46 & $<0.001$ \\
\hline Take Drug Again ${ }^{\mathrm{a}}$ & $82.0(28.4)$ & $37.0(29.0)$ & $34.5(24.2)$ & $<0.001$ & 0.42 & $<0.001$ \\
\hline Good Effects & $94.0(21.2)$ & $20.0(34.9)$ & $2.7(11.7)$ & $<0.001$ & 0.07 & $<0.001$ \\
\hline High & $94.6(21.3)$ & $19.6(33.4)$ & $2.9(11.7)$ & $<0.001$ & 0.05 & $<0.001$ \\
\hline Bad Effects ${ }^{\mathrm{b}}$ & $11.5(22.4)$ & $7.5(21.2)$ & $2.9(12.3)$ & - & - & - \\
\hline Feeling Sick ${ }^{\mathrm{b}}$ & $10.3(23.0)$ & $7.5(23.2)$ & $3.0(10.8)$ & - & - & - \\
\hline Drowsiness/Alertness $^{\mathrm{c}}$ & $32.1(25.1)$ & $47.3(23.4)$ & $47.2(25.0)$ & $<0.001$ & 0.91 & 0.04 \\
\hline
\end{tabular}

${ }^{a}$ End-of-treatment assessments

b Treatment differences were not assessed because overall treatment effect was not significant

${ }^{\mathrm{c}} E_{\min }$ values used for Drowsiness/Alertness

$E_{\max }$ maximum effect, $E_{\min }$ minimum effect, $O X Y$ oxycodone $0.07 \mathrm{mg} / \mathrm{kg}, s O X N$ simulated oxycodone $0.07 \mathrm{mg} / \mathrm{kg}+$ naloxone $0.035 \mathrm{mg} / \mathrm{kg}, S D$ standard deviation, VAS visual analog scale [either unipolar (i.e., $0=$ no effect) including High, Good Effects, Bad Effects or bipolar (i.e., $50=$ neutral response) including Overall Drug Liking, Take Drug Again, and Drowsiness/Alertness]

maximum pupil constriction of $1.1 \mathrm{~mm}$ (SD $0.7 \mathrm{~mm}$ ), which was similar to the maximum pupil constriction of 1.1 (SD $0.6 \mathrm{~mm}$ ) after placebo administration. The leastsquares mean difference was $-1.62 \mathrm{~mm}$ for placebo vs. OXY $(p<.001)$ and 1.59 for sOXN vs. OXY $(p<.001)$. No significant difference was found between placebo and sOXN.

\subsection{Safety and Tolerability}

The overall incidence of treatment-emergent adverse events (TEAEs) in the safety population $(n=23 / 24)$ after study drug administration was $20.8 \%$ with placebo, $29.2 \%$ with sOXN, and $95.7 \%$ with OXY. The most common TEAEs occurring during this study were euphoric mood, feeling hot, somnolence, and headache (Table 2).

There were two incidents of ventricular tachycardia, considered a serious TEAE. One event occurred following administration of sOXN, and the other after administration of placebo; the latter subject discontinued the treatment phase because of the TEAE. Both events were deemed medically important, mild in severity, and possibly related to study drug. These events resolved within $1 \mathrm{~min}$ without medical intervention. 
Fig. 5 Mean Good Effects VAS scores over time for OXY, sOXN, and placebo $(n=22)$. sOXN simulated oxycodone/ naloxone, $O X Y$ oxycodone, $V A S$ visual analog scale

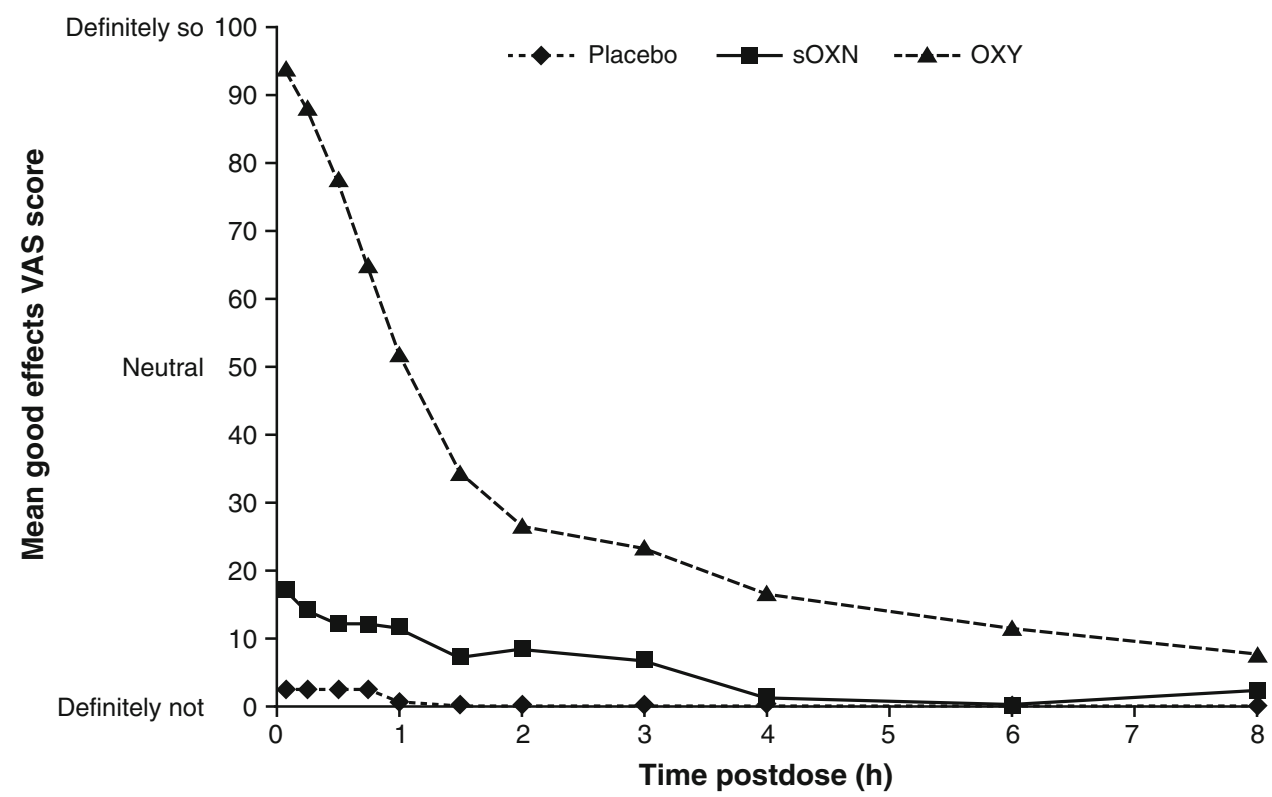

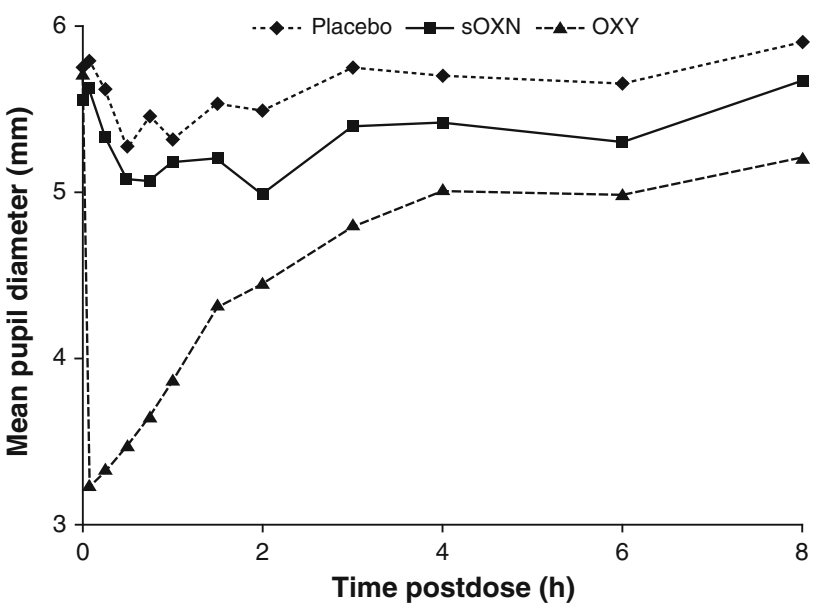

Fig. 6 Mean pupil diameter $(\mathrm{mm})$ over time for OXY, sOXN, and placebo $(n=22)$. (Pupil size measured at predose, $0.083,0.25,0.5$, $0.75,1,1.5,2,3,4,6$, and $8 \mathrm{~h}$ post-dose.) $s O X N$ simulated oxycodone/naloxone, $O X Y$ oxycodone

\section{Discussion}

Intravenous administration of oxycodone alone (OXY) or in combination with naloxone $(\mathrm{sOXN})$ resulted in similar plasma concentration profiles over time, but the pharmacodynamic effects, safety, and tolerability of sOXN were similar to placebo. Subjects experienced significantly less drug liking and other measures of abuse potential following administration of sOXN compared with OXY. These observations suggest that the coadministration of a $\mu$-receptor antagonist at the current ratio is sufficient to block the agonist effects of oxycodone. The lower abuse potential of the 2:1 oxycodone/naloxone of sOXN observed in this study is anticipated to contribute to a reduction in OXN abuse
Table 2 Adverse events occurring in $>5 \%$ of study subjects during assessment phase

\begin{tabular}{llll}
\hline Adverse event & $\begin{array}{l}\text { Placebo } \\
(n=24)\end{array}$ & $\begin{array}{l}\text { sOXN } \\
(n=24)\end{array}$ & $\begin{array}{l}\text { OXY } \\
(n=23)\end{array}$ \\
\hline Any & $5(20.8)$ & $7(29.2)$ & $22(95.7)$ \\
Feeling hot & $1(4.2)$ & $4(16.7)$ & $9(39.1)$ \\
Headache & $2(8.3)$ & $4(16.7)$ & $1(4.3)$ \\
Somnolence & $0(0)$ & $4(16.7)$ & $7(30.4)$ \\
Euphoric mood & $0(0)$ & $2(8.3)$ & $17(73.9)$ \\
Nausea & $0(0)$ & $1(4.2)$ & $2(8.7)$ \\
Dizziness & $0(0)$ & $0(0)$ & $3(13.0)$ \\
Pruritus & $0(0)$ & $0(0)$ & $3(13.0)$ \\
$\quad$ generalized & & $0(0)$ & $2(8.7)$ \\
Vision blurred & $0(0)$ & $0(0)$ & $2(8.7)$ \\
Pruritus localized & $0(0)$ &
\end{tabular}

Note: Values are expressed as $n(\%)$

$O X Y$ oxycodone $0.07 \mathrm{mg} / \mathrm{kg}$, sOXN simulated oxycodone $0.07 \mathrm{mg} /$ $\mathrm{kg}+$ naloxone $0.035 \mathrm{mg} / \mathrm{kg}$

liability in real-world settings. Because numerous complex factors affect real-world abuse liability, post-marketing epidemiologic studies will be needed to assess the actual impact of OXN's abuse deterrent properties.

This placebo- and active-controlled trial measured the pharmacokinetic and pharmacodynamic effects of intravenous administration of $\mathrm{sOXN}$ to characterize abuse properties in an opioid-experienced recreational population. Adhering closely to study-design guidance from the US FDA, recreational drug users were enrolled after confirmation of their ability to distinguish between placebo and an active compound. All subjects also passed the naloxone challenge test immediately prior to the treatment 
phase, indicating that they were not opioid dependent. A limitation of this study was the dose ratio selection. An oxycodone dose of $0.07 \mathrm{mg} / \mathrm{kg}$ (1-min infusion) was selected based on previous clinical experience in the same population [13]. Naloxone was administered at a dose of $0.035 \mathrm{mg} / \mathrm{kg}$ according to the intended OXN ratio of $2: 1$. However, this dose ratio may not represent the situation in real-world settings. Abusers likely crush tablets, extract oxycodone from combined tablets, and dissolve the extracts in an aqueous medium for a single intravenous administration, which may have higher or lower concentrations or different ratios than the study preparation, depending on the method of extraction. However, laboratory testing indicates that the inactivation of naloxone and the separation of oxycodone from naloxone are difficult to achieve (unpublished data).

Assessments of drug liking, subjective drug value, and other measurements proved valuable in assessing medications for potential misuse [2]. End-of-treatment and timecourse measurements of pharmacodynamic effects showed significant differences between $\mathrm{OXY}$ and sOXN administration, but not when sOXN was compared with placebo. Objective assessment by pupillometry supported these subjective responses, as well as the low incidence of euphoric mood associated with sOXN compared with OXY, indicating that naloxone antagonized the central activity of oxycodone. Low scores for Bad Effects VAS and Feeling Sick VAS for all treatments indicated minimal negative effects, as well. The current study suggests that sOXN has significantly fewer rewarding effects, which might make the tablet formulation less appealing to recreational drug users when crushed and dissolved for injection. Formulations that can be manipulated to produce a faster onset of action to quickly attain peak rewards have the highest likelihood for abuse [2].

Although the pharmacodynamic effects of sOXN were similar to placebo, sOXN and OXY demonstrated similar oxycodone exposures. Pharmacodynamic VAS scores after OXY administration had peak and duration of effects consistent with oxycodone concentration profiles. sOXN administration resulted in significant attenuation of oxycodone Drug-Liking VAS ratings compared with OXY. Overall, sOXN was well tolerated and the AE profile was similar to known effects of opioid agonists. The most common AEs following OXY and sOXN administration included euphoric mood, feeling hot, and somnolence, although the incidence of these events was lower after sOXN than OXY, and lowest following placebo administration. These results suggest that naloxone significantly reduces, but does not completely eliminate, the central effects of oxycodone when co-administered intravenously. The overall incidence of these effects may be affected by the central exposure to naloxone.
Prescription opioid analgesics are an important component of modern pain management. However, their abuse and misuse represent a serious and growing public health issue. The development of abuse deterrent opioid analgesic formulations represents one important approach to addressing this public health problem. [7]. Some recreational drug users report greater reinforcing effects following intravenous opioid administration, likely the result of the more rapid rise of drug concentrations in the central nervous system [7]. Intravenous administration of drugs is associated with increased health risks [6, 7]. An oxycodone formulation such as OXN that limits the reinforcing effects of oxycodone in the setting of intravenous abuse may, along with other efforts, lead to lower abuse liability in real-world settings. The present study demonstrated significantly lower drug-liking after sOXN, which is consistent with the agonist-antagonist interaction hypothesis. In addition to the present findings, we have shown in related research that $\mathrm{OXN}$ has reduced abuse potential following intranasal administration [16]. These findings suggest that the naloxone component of OXN confers meaningful abuse-deterrent properties in settings of intranasal and intravenous abuse [17].

Agonist/antagonist abuse-deterrent formulations have been used for other opioid agonists. For example, naloxone has been formulated with the partial opioid agonist buprenorphine (Suboxone) in a 4:1 ratio of buprenorphine to naloxone to decrease the abuse, misuse, and diversion of buprenorphine monotherapy. In one study, intramuscular injection of the buprenorphine/naloxone combination produced dose-related increases in opioid withdrawal in opioid-dependent individuals [18]. Similarly, another study demonstrated that subjects were less willing to pay for the intravenous buprenorphine/naloxone combination compared with either buprenorphine alone or heroin [19]. These results are consistent with the current study wherein participants were less willing to pay for the oxycodone/ naloxone combination compared with oxycodone alone. Nevertheless, the addition of naloxone might provide yet another deterrent to oxycodone abuse.

\section{Conclusions}

Significant reductions in the degree of drug liking and other pharmacodynamic measures after administration of sOXN compared with OXY indicate that naloxone concentrations were sufficient to antagonize the effects of oxycodone when abused by the intravenous route of administration in opioid-experienced drug users. Post-marketing epidemiologic data from a variety of sources will be needed to assess the effects of OXN's abuse deterrent properties on its abuse in real-world settings. 
Acknowledgments/Disclosures This study was sponsored by Purdue Pharma L.P. and conducted by INC Research Toronto. All authors were involved in the design of the study, data collection, analysis and interpretation, the development of this manuscript, and the decision to submit the manuscript for publication. All authors were full-time employees at their respective affiliations at the time the study was conducted. Mr. Perrino is currently affiliated with Regeneron Pharmaceuticals in Tarrytown, NY, USA, and Dr. Shram is currently affiliated with Altreos Research Partners, Inc, in Toronto, Ontario, CAN. The authors acknowledge Katherine Stevens, PhD, and Michael R. Brandt, PhD, of SCI Scientific Communications \& Information (Parsippany, NJ, USA) for providing editorial assistance with the development of the manuscript (funded by Purdue Pharma L.P.). The authors would also like to thank Henry Caporoso, MA, a full-time employee of Purdue Pharma L.P., for his assistance in preparing this manuscript.

Funding Funding for this study was provided by Purdue Pharma, L.P., Stamford, CT, USA.

Open Access This article is distributed under the terms of the Creative Commons Attribution Noncommercial License which permits any noncommercial use, distribution, and reproduction in any medium, provided the original author(s) and the source are credited.

\section{References}

1. OxyContin [package insert]. Stamford: Purdue Pharma L.P.; 2013.

2. US Department of Health and Human Services. Guidance for industry: abuse-deterrent opioids: evaluation and labeling. http:// www.fda.gov/downloads/Drugs/GuidanceComplianceRegulatory Information/Guidances/UCM334743.pdf. Accessed 19 March 2013.

3. Manchikanti L, Helm S, Fellows B, et al. Opioid epidemic in the United States. Pain Physician. 2012;15(3 Suppl):ES9-38.

4. Katz NP, Birnbaum H, Brennan MJ, et al. Prescription opioid abuse: challenges and opportunities for payers. Am J Manag Care. 2013;19(4):295-302.

5. Stanos SP, Bruckenthal P, Barkin RL. Strategies to reduce the tampering and subsequent abuse of long-acting opioids: potential risks and benefits of formulations with physical or pharmacologic deterrents to tampering. Mayo Clin Proc. 2012;87(7):683-94.

6. Butler SF, Black RA, Cassidy TA, Dailey TM, Budman SH. Abuse risks and routes of administration of different prescription opioid compounds and formulations. Harm Reduct J. 2011;8:29-45.

7. Budman SH, Grimes Serrano JM, Butler SF. Can abuse deterrent formulations make a difference? Expectation and speculation. Harm Reduct J. 2009;6:8-14.
8. Meissner W, Leyendecker P, Mueller-Lissner S, et al. A randomised controlled trial with prolonged-release oral oxycodone and naloxone to prevent and reverse opioid-induced constipation. Eur J Pain. 2009;13(1):56-64.

9. Smith K, Hopp M, Mundin G, et al. Low absolute bioavailability of oral naloxone in healthy subjects. Int J Clin Pharmacol Ther. 2012;50(5):360-7.

10. Dowling J, Isbister GK, Kirkpatrick CM, Naidoo D, Graudins A. Population pharmacokinetics of intravenous, intramuscular, and intranasal naloxone in human volunteers. Ther Drug Monit. 2008;30(4):490-6.

11. Sadock BJ, Sadock VA, Sussman N. Kaplan \& Sadock's pocket handbook of psychiatric drug treatment. 4th ed. Illustrated. Philadelphia: Lippincott Williams \& Wilkins; 2005.

12. Handelsman L, Cochrane KJ, Aronson MJ, Ness R, Rubinstein KJ, Kanof PD. Two new rating scales for opiate withdrawal. Am J Drug Alcohol Abuse. 1987;13(3):293-308.

13. Walsh SL, Nuzzo PA, Lofwall MR, Holtman JR Jr. The relative abuse liability of oral oxycodone, hydrocodone and hydromorphone assessed in prescription opioid abusers. Drug Alcohol Depend. 2008;98:191-202.

14. Griffiths RR, Troisi JR, Silverman K, Mumford GK. Multiplechoice procedure: an efficient approach for investigating drug reinforcement in humans. Behav Pharmacol. 1993;4(1):3-13.

15. Griffiths RR, Rush CR, Puhala KA. Validation of the multiplechoice procedure for investigating drug reinforcement in humans. Exp Clin Psychopharmacol. 1996;4(1):97-106.

16. Harris SC, Perrino PJ, Shram M, Bartlett C, Colucci SV, Wang Y. Abuse potential of oxycodone/naloxone (OXN) tablets administered intranasally in non-dependent recreational drug users with moderate opioid experience. Poster presented at American Society of Pain Educators' PAIN Week; 4-7 September 2013; Las Vegas. http://conference.painweek.org/media/mediafile_attach ments/00/650-painweek2013acceptedabstracts.pdf. Accessed 14 Apr 2014.

17. Wang Y, Perrino PJ, Schoedel KA, Geoffroy P, Colucci SV, Harris SC. Abuse potential of chewed or intact oxycodone/naloxone $(\mathrm{OXN})$ tablets in methadone-stabilized, opioid-dependent subjects when administered orally. Abstract presented at: American Society of Pain Educators' Pain Week; 3-6 September 2013; Las Vegas. http://conference.painweek.org/media/mediafile_ attachments/00/650-painweek2013acceptedabstracts.pdf. Accessed 14 Apr 2014.

18. Stoller KB, Bigelow GE, Walsh SL, Strain EC. Effects of buprenorphine/naloxone in opioid-dependent humans. Psychopharmacology (Berl). 2001;154(3):230-42.

19. Comer SD, Sullivan MA, Vosburg SK, et al. Abuse liability of intravenous buprenorphine/naloxone and buprenorphine alone in buprenorphine-maintained intravenous heroin abusers. Addiction. 2010;105(4):709-18. 